\title{
Narrative in der Eurokrise - Implikationen für die Wirtschaftspolitik
}

Jens Boysen-Hogrefe und Ulrich Stolzenburg

\section{Einleitung}

Die Eurokrise hat Konfliktlinien zwischen Europas Nationalstaaten aufbrechen lassen. Als Konfliktparteien werden immer wieder zwei Gruppen von Mitgliedsländern genannt: die Länder der Peripherie und Frankreich auf der einen Seite und die nördlichen Mitgliedsländer mit Deutschland auf der anderen Seite. Auf der einen Seite wird eine großzügigere Finanzpolitik gefordert, die - im Zweifel durch geldpolitische Maßnahmen flankiert - weitreichendere Handlungsspielräume erhalten soll. Auf der anderen Seite wird verlangt, die Geld- und Finanzpolitik an klaren Regeln auszurichten, vordringlich mit dem Ziel, zukünftige Krisen zu vermeiden. Während die eine Gruppe den Grund der Krise vor allem im Verhalten der volatilen Finanzmärkte sucht, denen der Staat ein starkes Gewicht entgegensetzen soll, sieht die andere Gruppe das Versagen des Staates selbst als wesentliche Ursache für die Krise. Auf den ersten Blick scheint die Konfliktlage leicht zu überblicken. Die Länder der Peripherie waren deutlich mehr von den direkten Folgen der Krise betroffen und verlangen nach Transfers und Schuldenvergemeinschaftung, die die Länder des Kerns bzw. im Norden nicht geben möchten. Die wirtschaftspolitischen Forderungen und die sie begleitenden Sichtweisen scheinen also schlicht eine Funktion des jeweiligen nationalen Interesses zu sein.

Doch das Bild, dass einzelne Länder als rational agierende Akteure schlicht nach ihrem ökonomischen Vorteil streben, ist schwer durchzuhalten. Brunnermeier, James und Landau argumentieren, dass es bei weitem nicht klar ist, wer von der Rettungspolitik profitiert. ${ }^{1}$ Deutsche Anleger hätten bei einem Ausbleiben von solchen Politikmaßnahmen sicherlich große Teile ihres Auslandsvermögens schnell verloren. Die nationalen Interessen sind mit Blick auf die Rettungspolitik für viele Mitgliedsländer zumindest nicht eindeutig zu benennen. Als weiterer Grund für 
die Aufgliederung der Konfliktparteien gelten ferner unterschiedliche Auffassungen über grundsätzliche Fragen der Wirtschaftspolitik sowie über die Rolle des Staates im Wirtschaftsgeschehen, was wiederum den Blick auf mögliche Ursachen und den Verlauf der Krise, also das Krisennarrativ bestimmt. Zum Teil scheinen die Auffassungen auf den jeweiligen wirtschaftspolitischen Traditionen zu fußen: Die deutsche Wirtschafts- und Krisenpolitik gilt als stark durch die ordoliberale Schule geprägt. ${ }^{2}$ Die französische Wirtschaftspolitik hingegen hat zumindest seit der Nachkriegszeit eher eine interventionistische Tradition. ${ }^{3}$

Ein Krisennarrativ kann verschiedene Funktionen erfüllen. Wie in der Medizin kommt die Diagnose vor der Therapie, und ohne eine zutreffende Diagnose kann eine zielgerichtete Behandlung kaum gelingen. Ein Krisennarrativ kann also als Grundlage verstanden werden, auf deren Basis sich geeignete Antworten zur Krisenbekämpfung (akut) und zur Krisenprävention (für die Zukunft) ableiten lassen. Aus dem Narrativ werden dann konkrete Handlungsanweisungen an die Wirtschaftspolitik abgeleitet. Sofern grundlegende Überzeugungen der Entwicklung eines gemeinsamen Europäischen Krisennarrativs im Wege stehen, dürfte es schwer fallen eine klare Antwort der Wirtschaftspolitik auf europäischer Ebene zu erreichen - häufig war vom `durchwurschteln die Rede. Brunnermeier, James und Landau fordern daher eine »Ideen-Union « für den gemeinsamen Währungsraum. ${ }^{4}$ Es bedürfe einer gemeinsamen wirtschaftspolitischen Grundorientierung. Dazu passend gibt es inzwischen unter international führenden Ökonomen durchaus gemeinsame Initiativen, um ein möglichst objektives, gemeinsames Krisennarrativ für die Schuldenkrise zu entwickeln, mit dem Ziel, die Wirtschaftspolitik im Euroraum effizienter und effektiver zu gestalten.

Politisch dient ein Narrativ jedoch nicht nur einer möglichst objektiven Aufarbeitung der Krisenursachen. Ein Narrativ ist eine Erzählweise über ein Thema, das geeignet ist, Werte und Emotionen zu transportieren und das für eine bestimmte Gruppe oder einen Kulturkreis sinn- und identitätsstiftend sein kann. Narrative können Rechtfertigung bieten und ein wichtiges Element im politischen Diskurs einer Demokratie sein. Schließlich sind die handelnden Politiker zum einen gehalten, sich ihren Wählern zu erklären, und zum anderen sind sie sicherlich versucht, den Stimmungen und vorherrschenden Narrativen zu entsprechen. So dient ein Krisennarrativ aus nationaler Perspektive wohl oft auch dazu, (1) Verantwortung für die Entstehung der Krise zu negieren, um dadurch (2) eigenen Handlungsdruck für politisch unpopuläre Maßnahmen zu reduzieren und (3) die ökonomische Anpassungslast bei anderen Ländern abzuladen. Selbst wenn die Gegenseite 
der Debatte dieses Narrativ nicht übernimmt und es somit im Rahmen der international auszuhandelnden Krisenantwort keine konkreten Folgen hat, kann eine Gruppe sich damit gegenseitig bestärken und sich selbst vergewissern und den sschwarzen Peter im Ausland verorten.

Hängt das Gedeihen oder gar der Fortbestand der derzeitigen europäischen Institutionen nun davon $\mathrm{ab}$, dass sich die Mitgliedsländer auf ein Krisennarrativ und somit einen gemeinsamen Blick auf die Wirtschaftspolitik einigen, weil sonst der immer noch andauernde Konflikt unlösbar wird? Oder kommt bei dieser Sichtweise dem Narrativ bzw. den mit ihm verbundenen wirtschaftspolitischen Grundideen ein zu großes Gewicht $\mathrm{zu}$ ? Im weiteren Text werden zunächst einige der konkurrierenden Narrative der Eurokrise vorgestellt und dann ihr Widerschein in der europäischen Krisenpolitik aufgezeigt. Im vierten Abschnitt wird dann am Beispiel der deutschen Wirtschafts- und Europapolitik der vermeintliche Einfluss der wirtschaftspolitischen Grundideen auf das tatsächliche wirtschaftspolitische Handeln diskutiert und schließlich werden daraus Schlussfolgerungen gezogen, welche dauerhafte Bedeutung ein heutiger Konsens über die Ursachen der Krise haben kann.

\section{Narrative in der Euro-Schuldenkrise}

Die Krise hat die Frage nach der Rechtfertigung des Euro-Projekts nach oben gespült. Solange die wirtschaftlichen Rahmendaten gut waren und zumindest in der großen Mehrheit der Mitgliedsländer die ökonomischen Perspektiven rosig, hat sich die Frage nach einer Rechtfertigung des Euro-Projekts gar nicht gestellt. Das Regelwerk wurde effektiv nicht hinterfragt. Angesichts der Krise, die viele >Verlierer und zumindest aus Sicht der Verlierer relative >Gewinner hervorgebracht hat, ist das Projekt Euro unter Rechtfertigungsdruck geraten und zugleich dem Verdacht ausgesetzt, dass das Regelwerk der Europäischen Union und besonders des Euros bzw. des Eurosystems dazu genutzt wird, die jeweils eigene Gruppe auf Kosten der jeweils anderen Gruppe zu übervorteilen. ${ }^{5}$

Bemerkenswerterweise haben sich Erzählungen in beiden Lagern, Süd wie Nord, Kern wie Peripherie, gebildet, die diese relativ extreme Sichtweise der gegenseitigen Übervorteilung und Dominanz beinhalten. Entsprechend wird auch die Verantwortlichkeit der Krise der jeweils anderen Gruppe zugeordnet. 
Die >deutsche< Perspektive, für die bespielhaft Buchtitel von Hans-Werner Sinn wie Die Targetfalle oder Gefangen im Euro ${ }^{6}$ stehen, lässt sich überspitzt wie folgt zusammenfassen: Deutschland bzw. der Kern haben sich mit falschen Versprechen bzw. Zusagen in die gemeinsame Währung treiben lassen. Die Länder der Peripherie haben die Vorteile der neuen gemeinsamen Institution EZB, die die Glaubwürdigkeit der geldpolitischen Institutionen des Kerns geerbt hat, gerne mitgenommen, aber kaum eine der zugesagten Anstrengungen unternommen, um strukturell Anschluss zu finden. In der Folge kam es $\mathrm{zu}$ einem Verlust an Wettbewerbsfähigkeit und einem Anschwellen der Verschuldung von privater und staatlicher Seite, die in diesem Maß nur möglich wurde, weil die gemeinsame noch glaubwürdige - Institution EZB für niedrige Risikoaufschläge sorgte, womit diese quasi wie eine Allmende von den Ländern der Peripherie ausgenutzt werden konnte. Schließlich wurden die Länder des Kerns auf verschiedenen Wegen in Haftung genommen und ihnen die Kosten der Krise somit aufgebürdet.

Die Gegenposition, für die beispielhaft Paul Krugman steht, ${ }^{7}$ sieht den Auslöser der Krise in Deutschland und schreibt einen Großteil der negativen Krisenfolgen der verfehlten Wirtschaftspolitik auf europäischer Ebene zu, die unter dem Druck deutscher Dominanz durchgesetzt wurde. Deutschland habe in den Jahren vor der Krise durch eine Politik der Lohnmoderation das europäische Wettbewerbsgefüge erschüttert. $^{8}$ In der Folge ist der Leistungsbilanzsaldo Deutschlands stark gestiegen, während der übrige Euroraum quasi genötigt war, das >billige Waren- und Kreditangebot aus Deutschland abzunehmen, da ein zügiger Preisausgleich in Ermanglung eines Wechselkurses ausblieb. Die Industrien in der Peripherie verkümmerten.

Diese beiden konkurrierenden Narrative könnten kaum weiter auseinanderliegen. Zudem fokussieren sie sich weniger auf die Krise als auf die Schuldfrage und die Institution der gemeinsamen Währung insgesamt, in der ein Vehikel der Ausübung wirtschaftspolitischer Dominanz des jeweils anderen Lagers vermutet wird. Die Krise ist somit das Aufbrechen eines schwerwiegenden Konstruktionsfehlers - nämlich eines ungleichmäßigen Machtgefüges - der Währungsunion, die durch die Krise in ihrer derzeitigen Form in Frage gestellt wird. Beide Narrative gehen sehr stark in eine Verteidigungshaltung. An der Krise ist das jeweils andere Lager schuld. Die Rolle eines Narrativs zur Selbstrechtfertigung wird an dieser Stelle

6 Vgl. Sinn, Hans-Werner: Die Targetfalle. Gefahren für unser Geld und unsere Kinder, München: Hanser 2012; Sinn, Hans Werner: Gefangen im Euro, München: Redline 2014.

7 Vgl. z.B. Krugman, Paul: »Being Bad Europeans«, in: The New York Times vom 30.11.2014, h ttps://www.nytimes.com/2014/12/01/opinion/paul-krugman-being-bad-europeans.html?_r=1 (letzter Abruf 20.06.2020).

8 Vgl. Bofinger, Peter: »Deutschland ist ein Vorbild für Europa«, in: Henning Meyer/Andrew Watt (Hg.), Die 10 Mythen der Eurokrise... und warum sie falsch sind, Düsseldorf/Berlin: IMK (Institut für Makroökonomie und Konjunkturforschung)/SE Publishing 2014, S. 47-58. 
deutlich. Ein gemeinsamer politischer Gestaltungsweg in Europa ist wohl kaum denkbar, wenn solche Positionen aufeinandertreffen. Der Euro als Geschichte der gegenseitigen Unterwerfung lässt sich kaum im Konsens erzählen, sondern bleibt wohl bei jeweiligen Schuldzuweisungen.

\section{Krise im Euroraum: Solvenz- gegen Liquiditätskrise}

Doch weisen auch Narrative, die nicht den Euro als Instrument der wirtschaftspolitischen Dominanz, sondern mehr die akute Krise fokussieren, große Unterschiede auf. Als Stereotypen unterscheiden Brunnermeier, James und Landau hier zum einen das Narrativ, das in der Krise vordringlich eine Solvenzkrise sieht, die durch das Missmanagement einiger Akteure entstanden sei, vor allem von staatlicher Seite (»Staatsversagen «). ${ }^{9}$ Durch das Fehlverhalten hätten sich Schulden aufgetürmt und einzelne Ökonomien hätten an Wettbewerbsfähigkeit verloren. In diesem Narrativ wird die Bedeutung von Anreizstrukturen betont und es wird vermutet, dass entsprechend Akteure aktiv waren bzw. sind, die nicht vollständig für ihr Tun verantwortlich gemacht werden konnten und können. Dies wiederum treibe diese dazu, sehr riskante Agenden zu verfolgen (»moral hazard«). Auf die aktuelle Krise bezogen könne insbesondere das Verhalten griechischer Politiker vor der Krise, durchgängig überhöhte Haushaltsdefizite in Kauf zu nehmen und dabei sogar Haushaltszahlen zu fälschen, beispielhaft genannt werden. Die wirtschaftspolitische Handlungsempfehlung, die einem solchen Narrativ folgt, strebt die Schaffung von Anreizstrukturen an, die »moral hazard « und »Zeitinkonsistenzprobleme« mindert. Folglich gelte es, das diskretionäre Verhalten insbesondere der Fiskalpolitik zu kontrollieren und an Regeln zu binden. ${ }^{10}$ Das Aufspannen von Rettungsschirmen würde hingegen $\mathrm{zu}$ weiterem Fehlverhalten anregen, da die Kosten des Fehlverhaltens zumindest teilweise nicht den Verantwortlichen entständen, sondern auf die Bereitsteller der Rettungsschirme abgewälzt würden.

$\mathrm{Da}$ in diesem Denken der Verantwortung eine zentrale Rolle zukommt, sollte die Wirtschaftspolitik die Eigenverantwortung stärken, also einen entsprechenden Ordnungsrahmen schaffen, und selber berechenbar sein. Dem freien Markt wird die Rolle zugewiesen, Informationen effizient verarbeiten zu können. Dies führe schließlich zu einer effizienten Allokation der Mittel, solange die Marktteilnehmer eigenverantwortlich handeln. Die Wirtschaftspolitik hingegen solle berechenbar sein, um den Marktprozess letztlich so wenig wie möglich zu stören. Eine starke

9 Vgl. Brunnermeier/James/Landau: The Euro.

10 Als Vorbild werden immer wieder Erfolge in der Celdpolitik ins Feld geführt, wo die Unabhängigkeit der Zentralbanken und deren selbst gewählte Bindung an Inflationsziele immer wieder als wesentlicher Grund für die Einhegung der Inflation in den jüngeren Jahrzehnten genannt wird. 
Regelbindung der Wirtschaftspolitik ist die konsequente Forderung. Im Bereich der Geldpolitik spielt diese Sichtweise auf die Wirtschaftspolitik unter den Stichworten >Unabhängigkeit der Zentralbank « und `Inflationsziek seit längerer Zeit eine wesentliche Rolle. Analog sollte aus dieser Sichtweise heraus auch die Finanzpolitik an feste Regeln gebunden werden. Die »No-Bail-Out«-Klausel wird als eine solche Regel aufgefasst und das Aufspannen der Rettungsschirme als Regelbruch, der den Markt in seiner Funktionsfähigkeit beeinträchtige.

Dieses Narrativ lässt sich ideengeschichtlich bei der Public Choice Theory und der Ordnungspolitik verorten. Das Auseinanderfallen von Handlung und Verantwortung wird hier grundsätzlich als zentrales Problem wahrgenommen. Im Fokus vieler Überlegungen dieser Schulen stehen daher vor allem staatliche Akteure, da diese typischerweise nicht persönlich haftbar für ihr politisches Tun sind bzw. ihnen langfristige Folgen ihrer Politiken nicht mehr selber, sondern zumeist späteren Politikergenerationen angelastet werden. Ferner können staatliche Institutionen durch Machtausübung die Kosten bestimmter Handlungen auf eigentlich Unbeteiligte abwälzen.

Im Kontrast zu dem Narrativ, das die Krise im Euroraum vordringlich als Solvenzkrise sieht, steht das Narrativ, das die Eurokrise vor allem als Liquiditätskrise erfasst. Im Unterschied zur Solvenzkrise sind die Probleme einer Liquiditätskrise nicht strukturell und dauerhaft angelegt, sondern vorübergehend, aber akut. In einer Liquiditätskrise besteht eigentlich genügend Vermögen, um alle Gläubiger zu bedienen, doch gibt es Probleme mit der Umsetzung in der Zeit. Im Fokus steht die Vorstellung, dass eine eigentlich beherrschbare Situation durch die mehr oder weniger plötzliche Verschiebung gewisser Rahmenbedingungen in eine krisenhafte Entwicklung falle, die erhebliche Kosten mit sich bringe, die aber eigentlich vermeidbar gewesen wäre. Ziel der Wirtschaftspolitik müsse es sein, auf die akute Situation derart einzuwirken, dass die krisenhafte Entwicklung ausbleibt oder gemindert wird.

Konkret entsteht eine Liquiditätskrise, wenn die Kapitalgeber bei einer eigentlich kaum veränderten Solvenzsituation des Schuldners nicht mehr bereit sind, diesen mit Krediten zu versorgen. In einer typischen Liquiditätskrise sieht sich das Unternehmen oder das Land kurzfristigen Zahlungsverpflichtungen gegenüber. Das eigene Vermögen ist aber langfristig gebunden oder erst über die Zeit abrufbar, so dass man auf Kredite angewiesen ist, deren Rückzahlung zu einem späteren Zeitpunkt aber eigentlich unproblematisch wäre. Wenn nun die Kreditgeber z.B. durch eine plötzliche Änderung der Risikowahrnehmung davon abgehalten werden, kurzfristig Kredite auszugeben, stünde ein eigentlich solventes Land vor der Zahlungsunfähigkeit. Sofern die wahrgenommene Gefahr einer Zahlungsunfähigkeit dann wiederum selber für Kosten - wie höhere Refinanzierungszinsen - sorgt, die wiederum das Vermögen angreifen, kann die Liquiditätskrise erst die befürchtete Solvenzkrise hervorrufen, gewissermaßen als selbsterfüllende Pro- 
phezeiung. Ziel der Politik sollte es hier sein, dem Aufkommen der Liquiditätskrise entgegenzuwirken. Das Aufspannen von Rettungsschirmen und das Eingreifen der Geldpolitik (»lender of last ressort«) sind in diesem Szenario die Mittel der Wahl.

In diesem Zusammenhang wird häufig von »Multiplen Zinsgleichgewichten« gesprochen. Demnach sind bei gleichen Fundamentaldaten des Schuldners die von den Kreditgebern geforderten Zinsen niedrig, solange alle darauf vertrauen, dass sich der Schuldner leicht bei anderen Kreditgebern refinanzieren kann. Die Gläubiger vertrauen also darauf, dass ihre Forderungen jederzeit von anderen Gläubigern übernommen würden. Kommen hier Zweifel auf, würden Gläubiger einen höheren Kreditzins (Risikoaufschlag) verlangen. Sind alle (potenziellen) Gläubiger skeptisch, steigen die geforderten Zinsen. Als bespielhaft für eine solche Situation gilt die Finanzkrise, in der mehr oder minder schlagartig nach der Pleite von Lehman Brothers die Kreditfinanzierung zwischen den Banken (Interbankenmarkt) zusammengebrochen ist. Die Krise des Euroraums wird im Zuge dieses Narrativs ähnlich wahrgenommen. Ausgehend vom (vielleicht tatsächlich) gefährdeten Griechenland hat sich die Risikowahrnehmung der Gläubiger schnell gewandelt und auch Länder, die zuvor nicht gefährdet waren, sahen sich steigenden Zinsforderungen gegenüber. Ohne die Griechenland-Erfahrung hätte es hier womöglich gar keinen Zinsanstieg gegeben. Wirtschaftspolitische Schlussfolgerung wäre, solchen Ansteckungseffekten zu begegnen und einen »lender of last resort « für Staatsschulden zu platzieren.

Dieses Narrativ weist Finanzmärkten eine zentrale Rolle in der Krisendynamik zu. Keynesianische und verhaltensökonomische Ansätze korrespondieren hiermit. Kern ist das Verhalten der Finanzmarktakteure, die demnach nicht über vollständige Information verfügen und daher auch Emotionen folgen und auf das Verhalten anderer Akteure reagieren (Herdenverhalten). Die Wirtschaftspolitik hingegen sollte sich ein diskretionäres Element bewahren, um in der Krisensituation effektiv handeln zu können.

\section{Rheingraben und Ideen-Union}

Die beiden Narrative lassen sich - wenn nicht an einzelnen Denkschulen, so doch an Denkrichtungen - im ökonomischen Diskurs festmachen. In den Verhandlungen zur europäischen Krisenpolitik weisen die deutschen Positionen vielfach Argumente auf, die deutlich vom Narrativ der Solvenzkrise beeinflusst sind. Feld, Köhler und Nientiedt konstatieren, dass die deutsche Krisenpolitik durchaus merklich von der Ordnungspolitik beeinflusst war. ${ }^{11}$ Im Gegensatz dazu weisen französische 
Initiativen und Argumente Bezüge zum alternativen Narrativ auf. Brunnermeier, James und Landau machen daher entlang dieser beiden Sichtweisen auf die Krise eine ideengeschichtliche Spaltung zwischen der Wirtschaftspolitik Deutschlands und Frankreichs aus, den sogenannten Rheingraben (»Rhine divide«). ${ }^{12}$ Letzterer wird auf eine historisch bedingte, ideengeschichtliche Spaltung zurückgeführt, die sich nun in der Krise offenbart habe und zum Problem geworden sei.

Die Hypothese, dass letztlich Unterschiede in der Ideengeschichte die Wirtschafts- und Krisenpolitik im Euroraum stark beeinflussen, wird durch die Komplexität der Krise selbst gestützt. Die Krise ist sehr facettenreich und bietet Beobachtungen, die beide Sichtweisen stützen. Die Finanzpolitik Griechenlands vor der Krise und das resultierende Ausmaß der Staatsschuld sind schwerlich allein mit den Termini einer Liquiditätskrise zu erfassen. Zugleich weisen zum Teil sehr plötzliche Veränderungen der Risikoeinschätzung der Marktakteure ausweislich der Renditen der an den Sekundärmärkten gehandelten Staatsanleihen auf die Existenz multipler Zinsgleichgewichte hin - vor der Krise waren bei zum Teil vergleichbaren Unterschieden der Fundamentaldaten die Renditen für nahezu alle Länder identisch, doch mit dem Beginn der Krise in Griechenland stiegen die Risikoaufschläge auch für andere Euroraumländer. ${ }^{13}$ Die Empirie ist somit nicht wirklich geeignet, eindeutig den Ausschlag für eines der beiden Krisennarrative zu geben.

Ebenso ist die schlichte Frage, was für den jeweiligen Nationalstaat von Vorteil ist, an was sich also die nationale Wirtschafts- und Krisenpolitik entsprechend orientieren sollte, kein Schlüssel zum Verständnis der widerstreitenden Positionen. So haben Rettungsschirme unter anderem auch deutsche Vermögen geschützt. Eine Liquiditätskrise, die auf viele Märkte übergreift, ist gerade nicht im Interesse der Gläubiger. Hingegen hätte zumindest aus heutiger Perspektive der Krisenverlauf für Griechenland womöglich weniger langwierig und drastisch erfolgen können, wenn das Land im Jahr 2010 die Rettungsschirme nicht in Anspruch genommen und den Staatsbankrott erklärt hätte.

Die Schlussfolgerung von Brunnermeier, James und Landau ist, dass die europäische Politik Einvernehmen über wirtschaftspolitische Grundfragen herstellen muss, um die Handlungsfähigkeit gegenüber Krisen zu bewahren und eine verlässliche Struktur des Währungsraums zu gestalten. ${ }^{14}$ Sie fordern, dass sich die

12 Vgl. Brunnermeier/James/Landau: The Euro.

13 Die Vertreter des Narrativs, das die Solvenzkrise in den Vordergrund stellt, argumentieren an dieser Stelle, dass die Marktteilnehmer vor der Krise trotz der Festlegung im MaastrichtVertrag bereits einen Bail-In erwartet haben und daher die Risikoaufschläge vor der Krise »künstlich « niedrig waren, vgl. Boysen-Hogrefe, Jens: »Für einen Schuldenschnitt und gegen den Rettungsschirm?«, in: Wirtschaftsdienst 91:7 (2011), S. 12-16. 
europäischen Partner in einen gemeinsamen Diskurs begeben, um ein gemeinsames Fundament der Ideen zu erlangen. Die Krise als ein prägendes historisches Ereignis könnte hierzu eine Chance liefern. In der Tat finden sich inzwischen Initiativen unter Beteiligung französischer und deutscher Ökonom/-innen, die ein gemeinsames Verständnis der Krise entwickeln, um daraus wirtschaftspolitische Empfehlungen herzuleiten. ${ }^{15}$

Eine wesentliche Erkenntnis dieser gemeinsamen Krisenanalysen ist, dass die enge Verbindung von finanzpolitischer und finanzwirtschaftlicher Stabilität - bzw. Instabilität - ein Brandbeschleuniger der Krise war (Bank-Sovereign-Loop bzw. Nexus von Staaten und Banken). So war die Aufgabe, eine systemische Finanzkrise zu verhindern, falls eine stark vernetzte Bank in Schieflage geraten sollte, implizit der jeweiligen heimischen Regierung zugeordnet. Dies führte dazu, dass ausufernde Bankenrettungen wie diejenigen im Zuge der Weltfinanzkrise auch die jeweiligen Staatsfinanzen in Bedrängnis bringen konnten (Beispiel: Irland). Gleichzeitig hatte diese stillschweigende staatliche Übernahme der Rolle des »lender of last resort« für das heimische Bankensystem zur Folge, dass die Bonität der heimischen Banken direkt von der wahrgenommenen Zahlungsfähigkeit des Staates abhängig war; im Falle einer Staatsschuldenkrise war die finanzielle Rückendeckung nicht mehr garantiert, da der finanzielle Rückhalt ja bereits selbst ins Wanken geraten war. Dieselbe Ansteckungsrichtung von den Staatsfinanzen auf die Finanzwirtschaft verstärkte sich zusätzlich, wenn Banken ein Klumpenrisiko in ihren Bilanzen aufwiesen, da sie häufig überproportional viele Anleihen des eigenen Staates hielten. Im Fall einer Staatsschuldenkrise schmilzt der Wert dieser Anleihen notwendigerweise zusammen, so dass die Kapitaldecke angegriffen wird und die Zahlungsfähigkeit der Bank ebenfalls bzw. zusätzlich in Zweifel geraten kann. Im Ergebnis dieser gegenseitigen Abhängigkeit droht eine Staatspleite den heimischen Bankensektor mit sich zu reißen, und da die EZB nur solvente Banken mit Liquidität versorgen darf, wäre eine Währungskrise unvermeidlich - das betreffende Land müsste über die Einführung einer eigenen Währung nachdenken. Eine Schlussfolgerung aus diesem Teufelskreis ist, dass der enge Zusammenhang von finanzpolitischer Solidität und finanzwirtschaftlicher Stabilität entflochten werden muss.

15 Vgl. Baldwin, Richard/Giavazzi, Francesco (Hg.): The Eurozone Crisis. A Consensus View of the Causes and a Few Possible Solutions, London: CEPR (Centre for Economic Policy Research) Press 2015; Bénassy-Quéré, Agnès/Brunnermeier Markus K./Enderlein, Henrik et al.: »Reconciling Risk Sharing with Market Discipline: A Constructive Approach to Euro Area Reform«, in: CEPR Policy Insight 91 (2018), S. 1-23. 


\section{Entsprechungen der Narrative in der europäischen Krisenpolitik}

Beide der im vorangegangen Abschnitt zunächst diskutierten Narrative haben Eingang in die europäische Krisenpolitik genommen. In der akuten Phase der Staatsschuldenkrise wurden Rettungsschirme von den Euro-Staaten aufgespannt. Im Jahr 2010 hat Griechenland ein Hilfspaket der anderen Mitgliedsländer (»Greek Loan Facility«) unter Mitwirkung des IMF erhalten. Später traten EFSM und EFSF auf den Plan, die schließlich vom ESM abgelöst wurden. Die Rettungsschirme wurden in einer Situation installiert, als - ausgehend von der Krise Griechenlands die Risikoaufschläge anderer Staaten ebenfalls stiegen. Ähnlich wie die Rettungsschirme für Banken in der Finanzkrise 2008/2009 sollte auch die Finanzhilfe für Griechenland dazu dienen, die Erwartungen auf den Finanzmärkten zu stabilisieren. Das Signal, dass die griechischen Anleihen ausdrücklich nicht ausfallen, war vordringlich an potenzielle Gläubiger anderer Staaten gerichtet, damit diese auch mit Staatsanleihen von Portugal, Irland, Spanien oder Italien ein niedrigeres Risiko verbinden würden. Im Blick waren dabei nicht nur die betroffenen Staaten, sondern ausdrücklich auch das europäische Banken- und Finanzsystem, das einen bedeutenden Teil der Staatsschuld der betroffenen Länder hielt.

Der Versuch, eine Ansteckung anderer Länder zu unterbinden, hat jedoch nicht in ausreichendem Maße verfangen. Nach Griechenland folgten weitere Rettungspakete für Portugal, Irland und abermals Griechenland. Erst das Einschreiten der EZB durch die Ankündigung des OMT-Programms, wonach diese notfalls unbegrenzt Staatsanleihen von in Bedrängnis geratenen Ländern aufkaufen könnte, führte schließlich zu einem allgemeinen Rückgang der Risikoaufschläge. Während bei den Rettungspaketen der Euroländer der Zweifel an der ausreichenden Finanzausstattung mitschwang, um auch große Mitgliedsländer wie Spanien oder Italien Finanzhilfe leisten zu können, konnte erst die EZB die Rolle des »lender of last resort« glaubwürdig ausfüllen. Es genügte die bloße Ankündigung, um den Stimmungsumschwung an den Anleihemärkten einzuleiten, ohne dass die EZB bislang auch nur eine einzige Anleihe unter dem OMT-Programm erworben hat. ${ }^{16}$

Diese Art der Krisenpolitik war getrieben von der Idee einer Liquiditätskrise, der Vorstellung, eigentlich solvente Länder bräuchten snur eine hinreichende Zwischenfinanzierung. Es schien somit geboten, den zeitweisen Vertrauensverlust an den Finanzmärkten zu überbrücken. Das Eingreifen der Staaten und der Zentralbanken stabilisierte die Märkte, während diese auf ein sschlechtes Zinsgleichgewicht zusteuerten, das letztlich mehrere Mitgliedsländer hätte in Bedrängnis bringen können. Das Bild eines Gleichgewichts ist zwar etwas schief, da steigende

16 Zum Einfluss von OMT auf die Anleihemärkte in Europa vgl. Boysen-Hogrefe, Jens: »Risk Assessment on Euro Area Government Bond Markets: The Role of Covernance Quality«, in: Journal of International Money and Finance 73 (2017), S. 104-117. 
und untragbar hohe Refinanzierungskosten offensichtlich keinen Gleichgewichtszustand darstellen. Gleichwohl hat der Eingriff der EZB letztlich geholfen, die akute Krise zu überwinden und ein Abgleiten in eine Solvenzkrise - jenseits vielleicht der Krise in Griechenland - zu verhindern.

Auf der anderen Seite wurde das europäische Regelwerk im Zuge der Krise mehrfach reformiert und verschärft, um Solvenzkrisen für die Zukunft vorzubeugen. Der Fiskalpakt sieht striktere Regeln für die Haushaltsdisziplin vor und zielt auf einen allmählichen Rückgang der öffentlichen Schuldenquoten ab. Das sogenannte Europäische Semester wurde eingeführt und nach wenigen Jahren nochmals reformiert. Das makroökonomische Ungleichgewichteverfahren brachte weitere Felder der Wirtschaftspolitik neben der Finanzpolitik unter die Aufsicht durch die Europäische Kommission. Schließlich wurde die Gläubigerbeteiligung in Rettungspakete für Griechenland und Zypern aufgenommen. Im Hinblick auf die Bankenregulierung wurden die Kapitalanforderungen schrittweise erhöht, um die Fähigkeit des Bankensystems zu verbessern, eintretende Verluste zu absorbieren. $\mathrm{Zu}$ dem wurden die Abwicklungsregeln für den Bankensektor grundlegend reformiert, so dass im Krisenfall zunächst private Gläubiger und Besitzer großer Bankeinlagen haftbar gemacht werden können. Schließlich wurden private Banken verpflichtet, darüber hinaus im Zeitverlauf einen Abwicklungsfonds aufzubauen, der im Fall einer Bankenschieflage zum Einsatz kommen soll, bevor staatliche Mittel herangezogen werden können. In vielen der genannten Punkte lässt sich das Narrativ einer Solvenzkrise wiederfinden. So soll z.B. die Einbeziehung der Gläubiger die Verantwortung der Marktakteure stärken und dem Markt wieder stärker die Rolle einer Kontrollfunktion zuweisen, die diesem unter dem Narrativ der Liquiditätskrise zumindest in der akuten Situation gerade abgesprochen würde.

Jenseits dessen sind weitere, substanzielle Veränderungen am institutionellen Rahmenwerk der Währungsunion derzeit nicht zu erwarten. Das hat verschiedene Gründe: Erstens steht das Krisenvermächtnis einiger Länder zusätzlichen Maßnahmen im Wege. Beispielsweise hat Italien mit seiner hohen öffentlichen Schuldenlast wohl kein Interesse daran, den Kreis der potenziellen Käufer seiner eigenen Staatsanleihen irgendwie einzuschränken. Somit dürfte das Privileg von Staatsanleihen in der Bankenregulierung nicht angetastet werden, wonach diese regulatorisch als ausfallsicher behandelt werden und folglich nicht mit Kapital unterlegt werden müssen. Auch Klumpenrisiken bei den Banken, welche oft überproportional viele Anleihen des eigenen Heimatlandes halten, können aus demselben Grund kaum reduziert werden. Zweitens ist der Leidensdruck nach einigen Jahren wirtschaftlicher Erholung und sinkenden Arbeitslosenzahlen wohl nicht mehr groß genug, um zusätzliche, weit reichende Reformen zu vereinbaren, die der Wählerschaft verkauft werden müssen. Politisches Kapital ist begrenzt, und ohne ein akutes Bedrohungsszenario können Widerstände gegen substanzielle Veränderungen kaum überwunden werden. Drittens sind einige diskutierte Maßnah- 
men häufig auch umstritten, da es dabei potenziell einfach um sehr viel Geld geht. Beispielsweise würde eine europäische Arbeitslosenversicherung milliardenschwere zwischenstaatliche Transfers ermöglichen, die je nach Ausgestaltung potenziell dauerhaft in eine Richtung laufen könnten. Eine gemeinsame Einlagensicherung für Bankguthaben würde eine grenzüberschreitende Haftung einführen, die naturgemäß nicht gleichermaßen im Interesse aller Beteiligten ist. Letztlich gibt es doch erhebliche Uneinigkeit bei der Frage, ob man sich in der langen Frist auf einen europäischen Superstaat zubewegen möchte (Fiskalunion, politische Union), oder aber ob Wirtschafts- und Finanzpolitik vollständig auf nationaler Ebene belassen werden sollen.

\section{Wie prägend sind die wirtschaftspolitischen Grundüberzeugungen für die Wirtschaftspolitik? - Die deutsche Erfahrung}

Das vorangegangene Kapitel hat belegt, dass es vor allem in der akuten Phase eine gemeinsame Krisenpolitik der europäischen Mitgliedsländer gegeben hat. Die Positionen beider Lager tauchen in den Einigungen und Beschlüssen auf. Auf der einen Seite wurden Rettungsschirme gespannt und mit dem ESM institutionalisiert, auf der anderen Seite wurden die Regeln für Finanzpolitik und die Finanzwirtschaft verschärft und neue Kontrollmechanismen für die Wirtschaftspolitik geschaffen.

Dieser Konsens war allerdings mit erheblichen Konflikten verbunden und hatte spürbare politische Kosten. Kosten, die vielleicht geringer gewesen wären, wenn es vorab einen stärkeren Konsens über Fragen der grundlegenden wirtschaftspolitischen Ausrichtung gegeben hätte. Doch wirft dies die Frage auf, welche Bedeutung zum Beispiel die ordnungspolitische Tradition tatsächlich für die Wirtschaftspolitik in Deutschland und für den deutschen Beitrag zur europäischen Wirtschaftspolitik hat.

Dazu nochmal ein Blick auf den »Rheingraben«. Überspitzt man dieses Bild, schreiben die Deutschen die Regeln, und die Franzosen brechen sie. Seit der Krise schien dieses Bild an Plausibilität zu gewinnen. Während Deutschland in jüngerer Zeit die Vorgaben des Stabilitäts- und Wachstumspakts einhielt, gelang dies Frankreich durchgängig nicht. Nicht früher als im Jahr 2018 lag das Budgetdefizit des Staates erstmalig unter der Marke von 3 Prozent in Relation zum Bruttoinlandsprodukt. Im Jahr 2019 stieg das Budgetdefizit wegen konjunktureller und politischer Entwicklungen wieder auf 3 Prozent. Das Erreichen der noch strikteren Vorgaben des Fiskalpakts liegt in weiter Ferne.

Die französischen Haushaltszahlen laden zur Interpretation ein, dass ein Vertrag unterzeichnet wurde, ohne die Intention, sich daran zu halten. Doch muss das vertragstreue Bild, das Deutschlands Staatshaushaltszahlen in den vergange- 
nen Jahren zeigen, nicht unbedingt Ausdruck einer stark an Regelbindung und Vertragserfüllung orientierten Politik sein. In den Jahren 1999 bis 2008 waren die deutschen Haushaltsdefizite genauso oft jenseits der 3 Prozent wie die französischen (vgl. Abbildung 2). Dass sich dies gerade nach der Krise 2008/2009 änderte (vgl. Abbildung 1), liegt auch an einer wirtschaftlichen Entwicklung, die durchgängig besser war als während der jeweiligen Haushaltsplanung erwartet. Ähnliches gilt mit umgekehrten Vorzeichen für die Zinsausgaben des Staates. Die selbst auferlegte Schuldenbremse konnte der deutsche Staat ohne größere Konsolidierungsanstrengungen einhalten. Der Bund hatte lediglich das so genannte »Zukunftspaket« im Jahr 2011 als Konsolidierungsmaßnahme zur Einhaltung der Schuldenbremse auf den Weg gebracht und es auch nur halbherzig umgesetzt bzw. umsetzen müssen. Die politischen Kosten einer an den Regelvorgaben gebundenen Politik waren praktisch nicht vorhanden.

Abbildung 1: Anzahl der Verstöße gegen das 3 \%-Kriterium 1999-2017.

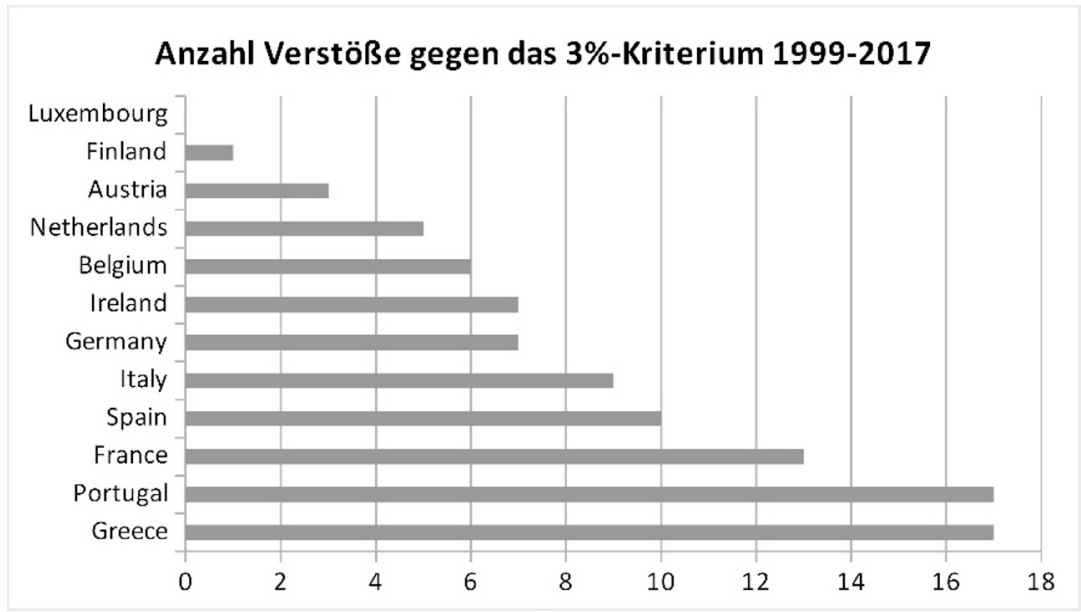

Quelle: Eurostat, eigene Berechnung und Darstellung.

Dass die deutsche Wirtschaftspolitik auch in jüngeren Jahren die Vorgaben aus Brüssel nicht $\mathrm{zu}$ intensiv verfolgt, sieht man hingegen an den wirtschaftspolitischen Empfehlungen, die im Rahmen des makroökonomischen Ungleichgewichteverfahrens getätigt werden. Deutschland liegt laut Alcidi und Gros bei der Zahl der nicht umgesetzten Empfehlungen in der Spitzengruppe. ${ }^{17}$ Dazu sei erwähnt, dass

17 Vgl. Alcidi, Cinzia/Gros, Daniel: How To Further Strengthen the European Semester? In-Depth Analysis Provided at the Request of the Economic and Monetary Affairs Committee, European Parliament, Brüssel: EGOV (Economic Governance Support Unit) 2017. 
Abbildung 2: Anzahl der Verstöße gegen das 3 \%-Kriterium 1999-2008.

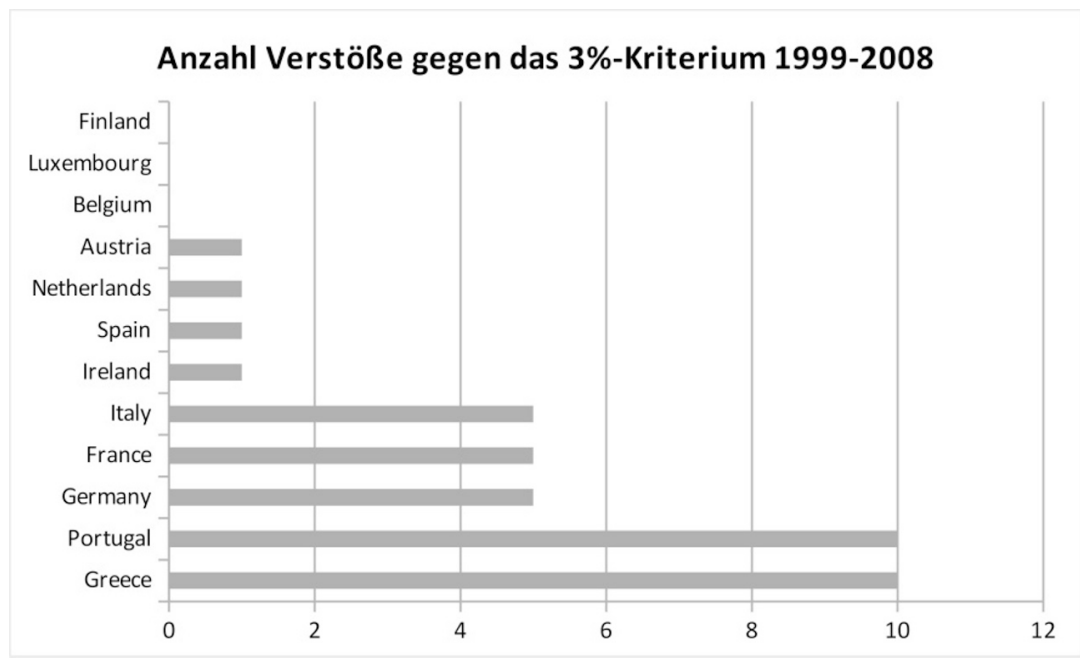

Quelle: Eurostat, eigene Berechnung und Darstellung.

viele der nicht umgesetzten wirtschaftspolitischen Empfehlungen, wie z.B. die Liberalisierung des Dienstleistungsmarkts durchaus in eine ordnungspolitische Agenda passen würden. Zwar sind die Empfehlungen aus dem Ungleichgewichteverfahren nicht im gleichen Maße verbindlich wie die Maastricht-Kriterien, doch deutet ihr Missachtung an, dass die deutsche Politik ähnlich stark oder schwach an europäischen Regeln ausgerichtet wird, wie es bei vielen anderen Staaten der Fall ist.

Dass möglicherweise viele der deutschen Ökonom/-innen ordoliberalen Ansätzen nahe stehen, ${ }^{18}$ bedeutet eben nicht, dass dadurch die Wirtschafts- und Europapolitik Deutschlands festgelegt sind. Wie weit die deutsche Wirtschaftspolitik mit Blick auf kurzfristige politische Überlegungen geht, zeigt vielleicht die Rettung des Baukonzerns Holzmann, die dem damaligen Kanzler Schröder durchaus

18 Vgl. Bofinger, Peter: »German Macroeconomics: The Long Shadow of Walter Eucken«, in: VoxEU/CEPR Policy Portal vom 07.06.2016, https://voxeu.org/article/german-macroeconomi cs-long-shadow-walter-eucken (letzter Abruf 20.06.2020). Döpke, Waldhof und Fritsche konstatieren angesichts einer von ihnen durchgeführten Befragung deutscher Makroökonom/innen anhand des Antwortmusters auf einige Fragen eine entsprechende Ausrichtung. Vgl. Döpke, Jörg/Waldhof, Gabi/Fritsche Ulrich: »Theories, Techniques and the Formation of German Business Cycle Forecasts: Evidence from a Survey of Professional Forecasters«, in: Journal of Economics and Statistics/Jahrbücher für Nationalökonomie und Statistik 239:2 (2019), S. 203-241. 
Sympathien eingebracht hat. Aus ordnungspolitischer Sicht ein klarer Fehltritt, der dann durch die später doch erfolgte Insolvenz als solcher bestätigt wurde. So weisen auch Feld, Köhler und Nientiedt darauf hin, dass die Wirtschaftspolitik in Deutschland nicht selten von ordnungspolitischen Grundsätzen abweicht. ${ }^{19}$

Um die deutsche Wirtschaftspolitik in der europäischen Schuldenkrise einzuordnen, ist ein Blick auf eine Umfrage des ifo Instituts informativ. Im Jahr 2016 haben Dolls und Wehrhöfer 2834 Personen in Deutschland über das Internet nach einer Bewertung der Transferpolitiken in Europa und Deutschland befragt. ${ }^{20}$ Während die Einstellung gegenüber Transferzahlungen auf europäischer Ebene sehr kritisch ist, ändert sich das Bild, wenn Transfers innerhalb Deutschlands bewertet werden. Die problematischen Anreizwirkungen von Transfers gelten allerdings sowohl im europäischen wie im nationalen Kontext. Kurz gesagt, hier deutet sich an, dass das Stimmungsbild in Deutschland in der Europa- und Krisenpolitik keinem festen wirtschaftspolitischen Kalkül entspringt, sondern von anderen Einflüssen etwa der Stärke von Gruppenidentitäten - dominiert wird.

Die Stimmungslage unter der Wählerschaft dürfte wiederum einen gewissen Einfluss auf die auf europäischer Ebene handelnden Politiker haben. Anhand der Beispiele darf bezweifelt werden, dass diese Stimmungslage stark an wirtschaftspolitischen Grundeinschätzungen orientiert ist.

Darüber hinaus dürfte selbst ein Konsens über die wirtschaftspolitischen Grundzüge unter Nicht-Politikern die Konflikte über die Ausgestaltung der Krisenpolitik nicht ausräumen. Dies konnte beispielhaft bei deutschen Wirtschaftswissenschaftler/-innen beobachtet werden, die der Ordnungspolitik nahestehen. Während Ottmar Issing, ehemaliger Chefvolkswirt der EZB, einen Schuldenschnitt für das angeschlagene Griechenland im Juni 2011 für katastrophal hielt, weil er den Schuldner aus der Verantwortung nehmen würde, haben die sogenannten Wirtschaftsweisen genau diesen Schuldenschnitt strikt gefordert, damit die Gläubiger mit in die Verantwortung genommen werden.

\section{Fazit}

Für den Fortgang des europäischen Projekts ist es sicherlich hilfreich, dass die politischen Berater über Ländergrenzen hinweg ins Gespräch kommen und an einem Konsens arbeiten, wie es bei den zuvor genannten Initiativen der Fall ist. Doch ist

19 Vgl. Feld, Lars P./Köhler, Ekkehard A./Nientiedt, Daniel: The >Dark Ages< of Cerman Macroeconomics and Other Alleged Shortfalls in German Economic Thought, Freiburger Diskussionspapiere zur Ordnungsökonomik, №. 17/03 (2017), S. 1-15.

20 Vgl. Dolls, Mathias/Wehrhöfer, Nils: Attitudes Towards Euro Area Reforms: Evidence from a Randomized Survey Experiment, EconPol Working Paper 2018/11. 
der Einfluss von Narrativen auf die politisch relevante Stimmungslage immer in Bewegung und Narrative selbst sind nicht unabhängig von Erfahrungswissen. Eine gemeinsame wirtschaftspolitische Position, die aus einer gemeinsamen Erzählung der Krise abgeleitet wird, ist daher wohl eher eine Momentaufnahme denn eine Garantie für eine dauerhafte und erfolgreiche Ausgestaltung der Wirtschaftspolitik in der Währungsunion.

Diese Skepsis gegenüber einer »Ideen-Union« soll nicht bedeuten, dass wirtschaftspolitische Grundüberlegungen und entsprechende Narrative keinen Einfluss hätten. Am Anfang der Währungsunion stand das Narrativ, dass der Euro den Ländern der Peripherie eine schnellere Konvergenz hin zu den Einkommensniveaus der Kernländer erlauben würde, was von Wirtschaftswissenschaftler/-innen als »Vehikeltheorie« bezeichnet wurde. Die Einführung einer gemeinsamen Währung sei demnach ein Vehikel, um die europäische Integration dauerhaft zu vertiefen. Dieses sehr optimistische Narrativ könnte Gläubiger und Schuldner verleitet haben, die Schuldentragfähigkeit öffentlicher wie privater Kreditnehmer zu überschätzen.

Die Skepsis gegenüber einer »Ideen-Union« ist somit auch kein Freibrief für wirtschaftspolitische Beliebigkeit. Das sehr ernsthafte Bestreben vieler europäischer Ökonom/-innen, gemeinsam Lehren aus der Krise zu ziehen und Lösungen für die Krise und die strukturellen Fragen der Währungsunion zu finden, beinhaltet substantielle Ideen, die den Euroraum krisenfester machen könnten. Doch bleibt unklar, was aus einem jetzigen Konsens im Fall einer nächsten Krise würde, wenn die Akteure - möglicherweise in ganz anderen Koalitionen zusammengewürfelt - den dann für sie relevanten Gegebenheiten ausgesetzt wären. Stimmungen und politische Mehrheiten könnten anders verteilt sein und dies dürfte die handelnden Politiker in einer solchen Situation mehr beeindrucken als ein zwischenzeitlicher Konsens.

Damit die »Ideen-Union« Wirklichkeit wird und kein bloßer Vorübergang bleibt, bedürfte es wohl Wählerinnen und Wähler, die sich als europäische Bürgerinnen und Bürger verstehen, und ein gerüttelt Maß ökonomischer Bildung. In der Zwischenzeit bleibt die Hoffnung, dass die Krise den Euroraum zwar auf eine harte Probe gestellt hat, aber er unter den Bedingungen des >Durchwurschtelns bisher überlebensfähig war - und bleibt.

\section{Literatur}

Ademmer, Esther/Boeing-Reicher, Claire/Boysen-Hogrefe, Jens/Gern, KlausJürgen/Stolzenburg, Ulrich: Euro-Area Fiscal Stance: Definition, Implementation and Democratic Legitimacy. In-Depth Analysis Provided at the Request 
of the Economic and Monetary Affairs Committee, European Parliament, Brüssel: EGOV (Economic Governance Support Unit) 2016.

Alcidi, Cinzia/Gros, Daniel: How To Further Strengthen the European Semester? In-Depth Analysis Provided at the Request of the Economic and Monetary Affairs Committee, European Parliament, Brüssel: EGOV (Economic Governance Support Unit) 2017.

Baldwin, Richard/Giavazzi, Francesco (Hg.): The Eurozone Crisis. A Consensus View of the Causes and a Few Possible Solutions, London: CEPR (Centre for Economic Policy Research) Press 2015.

Bénassy-Quéré, Agnès/Brunnermeier Markus K./Enderlein, Henrik et al.: »Reconciling Risk Sharing with Market Discipline: A Constructive Approach to Euro Area Reform«, in: CEPR Policy Insight 91 (2018), S. 1-23.

Bofinger, Peter: »Deutschland ist ein Vorbild für Europa«, in: Henning Meyer/Andrew Watt (Hg.), Die 10 Mythen der Eurokrise... und warum sie falsch sind, Düsseldorf/Berlin: IMK (Institut für Makroökonomie und Konjunkturforschung)/SE Publishing 2014, S. 47-58.

Bofinger, Peter: "German Macroeconomics: The Long Shadow of Walter Eucken «, in: VoxEU/CEPR Policy Portal vom 07.06.2016, https://voxeu.org/article/germa n-macroeconomics-long-shadow-walter-eucken (letzter Abruf 20.06.2020).

Boysen-Hogrefe, Jens: »Für einen Schuldenschnitt und gegen den Rettungsschirm?«, in: Wirtschaftsdienst 91:7 (2011), S. 12-16.

Boysen-Hogrefe, Jens: »Risk Assessment on Euro Area Government Bond Markets: The Role of Governance Quality«, in: Journal of International Money and Finance 73 (2017), S. 104-117.

Brunnermeier, Markus K./James, Harold/Landau, Jean-Pierre: The Euro and the Battle of Ideas, Princeton/Oxford: Princeton University Press 2016.

Bulmer, Simon: »Germany and the Eurozone Crisis: Between Hegemony and Domestic Politics«, in: West European Politics 37:6 (2014), S. 1244-1263.

Dolls, Mathias/Wehrhöfer, Nils: Attitudes Towards Euro Area Reforms: Evidence from a Randomized Survey Experiment, EconPol Working Paper 2018/11.

Döpke, Jörg/Waldhof, Gabi/Fritsche Ulrich: »Theories, Techniques and the Formation of German Business Cycle Forecasts: Evidence from a Survey of Professional Forecasters «, in: Journal of Economics and Statistics/Jahrbücher für Nationalökonomie und Statistik 239:2 (2019), S. 203-241.

Feld, Lars P./Köhler, Ekkehard A./Nientiedt, Daniel: „Ordoliberalism, Pragmatism and the Eurozone Crisis: How the German Tradition Shaped Economic Policy in Europe«, in: European Review of International Studies 2:3 (2015), S. 48-61.

Feld, Lars P./Köhler, Ekkehard A./Nientiedt, Daniel: The >Dark Ages` of German Macroeconomics and Other Alleged Shortfalls in German Economic Thought, Freiburger Diskussionspapiere zur Ordnungsökonomik, No. 17/03 (2017), S. 115. 
Krugman, Paul: »Being Bad Europeans«, in: The New York Times vom 30.11.2014, https:/www.nytimes.com/2014/12/o1/opinion/paul-krugman-bein g-bad-europeans.html?_r=1 (letzter Abruf 20.06.2020).

Sinn, Hans-Werner: Die Targetfalle. Gefahren für unser Geld uns unsere Kinder, München: Hanser 2012.

Sinn, Hans-Werner: Gefangen im Euro, München: Redline 2014. 